\title{
A formative evaluation of a staff reward and recognition programme
}

\author{
Authors: \\ Saleemah Salie ${ }^{1}$ \\ Anton Schlechter ${ }^{1}$ \\ Affiliations: \\ ${ }^{1}$ Section of Organisational \\ Psychology, University of \\ Cape Town, South Africa \\ Correspondence to: \\ Anton Schlechter \\ Email: \\ anton.schlechter@uct.ac.za

\section{Postal address} \\ Section of Organisational \\ Psychology, University of \\ Cape Town, Rondebosch \\ 7701, South Africa \\ Dates: \\ Received: 05 Oct. 2011 \\ Accepted: 17 Feb. 2012 \\ Published: 13 July 2012 \\ How to cite this article: \\ Salie, S., \& Schlechter, \\ A. (2012). A formative \\ evaluation of a staff \\ reward and recognition \\ programme. SA Journal \\ of Human Resource \\ Management/SA Tydskrif vir \\ Menslikehulpbronbestuur, \\ 10(3), Art. \#422, 11 pages. \\ http://dx.doi.org/10.4102/ \\ sajhrm.v10i3.422
}

(C) 2012. The Authors.

Licensee: AOSIS

OpenJournals. This work

is licensed under the

Creative Commons

Attribution License.
Orientation: It is generally assumed that reward and recognition programmes have increased staff motivation and reduced staff turnover.

Research purpose: The main aim of this evaluation was to test the plausibility of the programme theory underlying a staff reward and recognition programme within a retail setting. Secondary aims were to assess whether or not the programme was implemented as intended and whether or not its outcomes were well defined.

Motivation for the study: Different groups of people may have different assumptions about whether a reward and recognition programme works or not. This evaluation was motivated by the different assumptions held by programme stakeholders, programme recipients and social science researchers regarding the programme.

Research design, approach and method: This formative evaluation used a descriptive design. Primary qualitative data were collected by means of structured interviews with the Human Resource Development (HRD) Facilitator and ten programme participants.

Main findings: The results showed that the programme theory was not plausible and that the programme was not implemented as intended. Although the HRD Facilitator and the participants agreed that the programme led to improved customer service, they disagreed about the other programme outcomes.

Practical/managerial implications: This evaluation contains practical suggestions for improving the programme theory, the programme implementation process and the redefinition of the outcomes of the programme as standard performance indicators.

Contribution/value-add: This evaluation contributed to the limited literature on the effect of reward and recognition programmes. Whilst there is a vast amount of literature pertaining to such programmes, very few formal evaluations exist about them.

\section{Introduction}

The programme under evaluation was a staff reward and recognition programme implemented by one of the leading fashion retailers in South Africa. The evaluation aimed to explore whether or not the theoretical assumptions underlying this programme were plausible, whether or not it was implemented as intended and whether its outcomes were well defined or not.

Evaluators such as Chen (2005) and Rossi, Lipsey and Freeman (2004) have, in recent years, expounded on the merits of defining evaluations and developing guidelines which will assist in differentiating one evaluation study from another. An evaluation framework which allows evaluators to differentiate between various types of evaluation was used here (see Figure 1).

In terms of the framework presented above a theory evaluation can either focus on the programme theory and design element, or alternatively encompass an evaluation of the theory across the different elements proposed in the model. Chen (2005) indicates that theory evaluations are both descriptive and prescriptive by nature. A descriptive theory evaluation uncovers causal processes within the design, whilst a prescriptive theory evaluation examines the programme's composition in terms of generally accepted standards.

This evaluation assessed the programme theory across its design, implementation and proximal outcomes and was both descriptive and prescriptive by nature. The evaluators employed an inductive approach which allowed for the exploration of theory generated from actual programme activities and intended outcomes (Patton, 1997). Finally, the evaluation was formative, suggesting that evaluation results will be used for programme improvement (Trochim, 2006). 


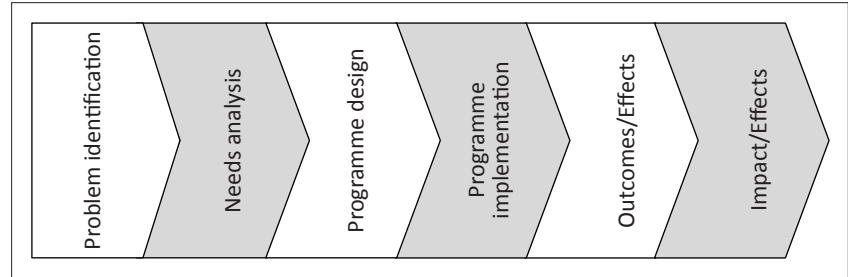

FIGURE 1: A framework of evaluation types.

\section{Prescriptive programme theory for reward and recognition programmes}

From the literature presented here it can be assumed that reward and recognition programmes increase motivation and reduce staff turnover.

\section{Increasing motivation}

According to Armstrong (2002), motivation refers to the factors which influence human behaviour and is usually comprised of three main elements: direction, effort and persistence.

One of the most common theories outlining the relationship between reward and motivation is Vroom's (1964) expectancy theory. This theory postulates that employees tend to be motivated when they receive rewards in exchange for doing their jobs, provided that these rewards are valued (Armstrong, 2002).

Motivation can be intrinsic or extrinsic (Armstrong, 2002). Intrinsic motivation refers to the '... inherent tendency to seek out novelty and challenges, to extend and exercise one's capacities, to explore, and to learn' (Ryan \& Deci, 2000, p. 70). In other words, individuals are motivated by self-generated factors such as pride, ownership, self-actualisation and the desire to grow and develop. On the other hand, extrinsic motivation refers to '... what is done to or for people to motivate them' (Armstrong, 2002, p. 56). This can include rewards such as increased pay, praise or promotion, as well as punishments, such as disciplinary action, withholding pay, or criticism. Each type of motivation has a different effect on human behaviour. Intrinsic motivation operates by means of self-motivation whilst extrinsic motivation is based on the expectation of recognition and reward (Jensen, McMullen \& Stark, 2007).

There is a vast amount of research on the effects of rewards on motivation and, whilst some theorists propose that rewards may have a detrimental effect on motivation (Applebaum, 1993; Deci \& Ryan, 1985; Kohn, 1993), there are others who advocate the use of rewards as effective tools to increase motivation (Cameron \& Pierce, 1996; Eisenberger \& Cameron, 1996; Nelson, 1998; Platten, 1996).

Although this controversial relationship between reward and recognition programmes and extrinsic and intrinsic motivation does exist, research suggests that if staff perceive a clear link between excellent performance and reward, the programme will indeed increase motivation. Such reward programmes would reinforce the employees' perceptions of their competence and would influence intrinsic motivation (Ulrich \& Brockbank, 2005).

According to Baker (1993), when organisations reward desired behaviours they reinforce particular outcomes and goals which may be strategically aligned to the organisation's vision and mission. For example, should the organisation value teamwork, then the reward and recognition scheme should be designed in such a way that outstanding teamwork is rewarded and positively reinforced.

Therefore, the underlying premise is that whilst rewards may indeed be used to increase motivation, motivation can only be sustained if both intrinsic and extrinsic motivational factors are incorporated in the programme design (Amabile, 1993). Mottaz (1985) indicates that the job level may serve as a moderating variable between reward and motivation: intrinsic reward showed a stronger relationship with motivation and job satisfaction in upper-level occupations, whilst extrinsic reward was related positively to motivation and job satisfaction in lower-level occupations.

\section{Reducing turnover}

Reward and recognition programmes have also been linked to a reduction in staff turnover. The concept of job commitment, which has been defined as '... the likelihood that an individual will stick with a job, and feel psychologically attached to it' has been linked directly to turnover (Rusbult \& Farrell, 1983, p. 430). Research showed that job commitment increases with an increase in job rewards and a reduction in turnover. Of interest here is that this relationship is evident from the start of the employment contract, which suggests that reward programmes may have a significant effect on the job commitment of employees as early on as the hiring phase (Rusbult \& Farrell, 1983). Reward programmes also influence turnover in the long term, as such programmes strengthen the incentive to retain excellent performers whilst generating an incentive for poor performers to leave (Bishop, 1986).

Harrison, Virick and William (1996) found that reward programmes produce greater job satisfaction, which reduces the desire for employees to consider alternative employment opportunities, and this, resultantly, reduces turnover. However, they further argue that the degree of reward contingency affects the performance-turnover relationship, which suggests that in order to reduce turnover schemes should be designed in such a way that rewards are not maximally contingent but rather linked to a combination of reward and recognition (Harrison et al., 1996).

In summary, reward programmes can be used to reduce staff turnover provided that such programmes include an element of intrinsic motivation and are reviewed throughout the employment contract in order to sustain performance.

\section{Opposing perspectives}

In conducting a theory evaluation, it is appropriate to consider different perspectives regarding the programme theory. For this reason the evaluators examined the views of critics who question the relationship between these programmes, motivation and staff retention. 
Firstly, Kohn (1993) provides a detailed analysis of why reward programmes cannot be relied upon to motivate employees or improve performance. He suggests that these programmes secure only temporary compliance and cannot guarantee long term results. He also argues that money does not necessarily motivate employees, as suggested by Hertzberg's (1959) research on motivating factors in organisations. This view is supported by AccelTeam (2008), a consulting company, which compared motivating factors and found that money did not rate very highly. Instead, the potential to grow in the company, having a job which is fulfilling and which has reasonable expectations and challenges, and identification with the organisation were stronger predictors of motivation.

Secondly, rewards are often viewed as punishment because of their manipulative nature (Deci \& Ryan, 1985; Kohn, 1993). Reward programmes may have unintended consequences like demoralisation, interpersonal tension and unhappiness for staff who are not rewarded. Kohn also indicated that individually based reward programmes could discourage the idea of teamwork and group participation.

Thirdly, and perhaps most importantly, it is suggested that reward programmes undermine the idea of intrinsic motivation (Kohn, 1993). Rewards are typically seen as a poor substitute for genuine feelings of accomplishment and satisfaction. Denning (2001) suggests that reward programmes may create a culture where employees expect to be rewarded for behaviour and performance that are considered as basic under normal circumstances.

Throughout the debate about the effects of reward and recognition on motivation, various meta-analyses have been conducted to investigate this assumed causal relationship. From Rummel and Feinberg (1988), through to Wiersma (1992) and Tang and Hall (1995), noteworthy results have indicated that rewarding performance with extrinsic rewards does indeed lower motivation. The explanation for this is the perceived controlling effect of extrinsic rewards.

However, literature also suggests that in specific industries, such as trade, the failure to reward performance will result in organisations losing their best employees (Beer, 1993). Beer maintains that incentive programmes encourage selfinterest and discourage organisational commitment. $\mathrm{He}$ suggests that employees should be paid equally, but that the top bracket of performers should additionally be rewarded for performance. This will allow the organisation to identify poor performers and recognise excellent performers.

Amabile (1993) and Baker (1993) state that there are both advantages and disadvantages associated with reward programmes. Amabile (1993) is of the opinion that incentive programmes stifle commitment and may also undermine intrinsic motivation. However, if rewards are perceived as providing new possibilities they may lead to an increase in creativity. Tangible rewards plus intrinsic motivation may be a good motivational combination. Baker indicated that reward programmes may have many unintended outcomes, but that well-designed reward programmes may have the desired effects.

In summary, it would seem that critics of reward programmes agree that if these programmes are well designed and implemented they may offer benefits like improved staff retention, employee effectiveness and loyalty. However, manipulative reward programmes cannot guarantee longterm goals and may discourage organisational commitment. Thus, alternative approaches, such as work process reorganisation, the need for training and the promise of promotions have been identified as substitutes for rewardbased programmes.

\section{Standards for reward and recognition programmes}

One key point has emerged thus far from the literature on such programmes, namely that reward programmes increase performance in the short term (Bowen, 2000). In this section the focus is on the design and implementation of an effective reward programme.

In order to understand reward, one has to understand motivation. Intrinsic motivation is internalised by the individual whilst extrinsic motivation is dependent on expectations, as discussed previously. Rewards can be offered as motivating factors provided that they are aligned with certain guidelines.

Firstly, concerning these guidelines, employees respond to reward out of a sense of personal choice. In other words they need to value the reward being offered. Furthermore, different factors motivate different people and therefore different people will value a specific reward differently (Morrall, 1996). A reward programme, tailored to different needs will have the desired effect.

Secondly, successful reward programmes incorporate both extrinsic and intrinsic motivators (Bowen, 2000). This follows from the first guideline: namely that whilst certain employees may value a higher base pay, others may be motivated by feedback and the opportunity to grow and develop.

Thirdly, organisational culture is reflected in a reward programme as it indicates which behaviours are important and valued by the organisation. For example, in a highperformance culture excellence may be rewarded and a mind-set will prevail where extraordinary effort is valued. Alternatively, if an organisation's focus is on customer service it makes sense that teamwork, consideration and regard for customers are top priorities (Bowen, 2000).

Fourthly, successful reward and recognition programmes need to be aligned with corporate goals (Benardin, 2003; Bowen, 2000). Programmes should be designed in such a way that performance measures are compatible with strategic goals for the long and short term. For instance, one would 
expect an organisation that designs new technology to have performance measures, which reward innovation (shortterm improvements to existing products) and creativity (a longer-term measure which rewards the design of new products). Both corporate goals and performance indicators should be measurable, clear, unambiguous, achievable, challenging and relevant to every person participating in the reward programme (Jensen et al., 2007; Zenger, 1992). This will provide direction to employees and clarify what they are striving towards.

Fifthly, and in line with expectancy theory (Vroom, 1964), employees must believe that an increase in performance will lead to a valued reward (Benardin, 2003; Zachary \& Kuzuhara, 2005). Should this element be present, then employees will be motivated to perform, with the understanding that performance will be rewarded.

In summary, the standards for an effective reward and recognition programme identified from the literature are that:

- the reward is tailored to individual needs

- it contains intrinsic and extrinsic motivators

- it reflects the company's culture

- it is aligned with its goals

- the recipients perceive a clear link between improved performance and reward.

\section{Description of the programme under evaluation}

The programme under evaluation was developed in a large retail organisation. It has been implemented in all 400 stores over the past five years. During the particular year of the evaluation the programme was being run for the fifth time. Small, non-significant changes are made to the programme on an annual basis. The programme is discussed and developed during the first half of the year and is launched officially in July.

The programme is a national staff reward and recognition programme based on a point system. The intervention is run annually for a six-month period. According to the stakeholders, reward and recognition principles have been used in its conceptualisation. It was designed by the Chief Executive Officer, the Human Resource Manager and the Marketing Department. The short-term programme goal is to provide outstanding customer service and motivate staff to drive their own development. The longer-term goals are to sustain motivational levels, encourage staff to perform at higher levels than those contained in their job descriptions, and to retain staff.

The target population comprises all employees at store level. This includes store managers, supervisors and staff at all branches. The programme is compulsory for the target population.

Guidelines for the programme are discussed annually in June and communicated to area and store managers, who in turn are required to launch and communicate the programme to employees. Each store receives a budget for its launch, which may consist of a launch breakfast, canteen decorations, or a morning of discussion, activities and games.

Each store receives a work chart with the names of its employees. The chart monitors and maps out the points awarded to employees for the remainder of that year. Points are also monitored on the organisational database, and tallied in December. Every month each store is awarded a certain number of points, according to particular measures such as turnover, customer feedback and the number of new accounts opened. These points are then divided equally amongst staff in that store and added to the points on the chart. Thus, the store receives the credit as a team. The number of points awarded to each store is decided by Head Office.

In terms of turnover, points are awarded when stores reach a certain percentage of their target each month (not divulged to the evaluators). Customer service is measured by contacting a randomly selected group of store customers who are required to rate the service they received. The number of new accounts opened per store is measured against the number of new accounts opened during the previous year.

There is also an opportunity for individual employees to score additional points. If an area or store manager recognises that certain individuals are excelling at customer service or are performing above their job descriptions, they communicate this to the human resource (HR) Department at Head Office where the points are added to the staff member on the store's chart.

During the intervention employees are also required to complete two staff workbooks which can earn them additional points. Whilst these workbooks are designed annually, they generally cover topics such as administrative aspects of the business, business culture, vision, strategy, health and safety and employee relations. The workbooks may also include areas of specific concern, namely stock loss and absenteeism. The workbooks are to be completed and returned within a three month period after which points are awarded.

Employees are able to check their scores by sending a short message service (SMS) to the database. This allows them to monitor points needed to reach the target and encourages them to strive for a higher point score. In December of each year, all work chart points are tallied nationwide. The HR Department will then select the top twenty-five employees across all 400 stores in South Africa, who have earned the most points. These individuals consequently receive a threeday holiday paid for in full by the organisation.

\section{Programme theory}

The HR Manager provided the following information on how the programme will change the recipients' behaviour (see Figure 2). 
The descriptive assumption or the underlying logic of the programme is that the intervention, which is made up of the activities described earlier, will lead to an increase in employee motivation, which in turn will lead to improved performance. This constitutes the causal theory underlying the intervention, or in evaluation terms, the cause-and-effect sequence (Rossi et al., 2004).

It is important to note that whilst the organisation considered the programme capable of achieving various other goals and objectives, such as employee retention and improved customer service, as discussed previously, these outcomes do not appear in the stakeholders' programme theory.

\section{Evaluation questions}

A formative evaluation improves a programme by examining its delivery, the quality of its implementation and the assessment of the organisational context, personnel procedures and inputs (Trochim, 2006). The primary goal of a formative evaluation is to collect feedback from programme recipients and stakeholders in order to make revisions where necessary (Bhola, 1990). The advantage of this on-going, dynamic process is that findings can be used to improve real-time programme activities and facilitate learning and effectiveness in the organisation (Chappuis \& Chappuis, 2008).

In essence then, the goals of a formative evaluation are:

- to provide rapid feedback

- to assist in documenting the programme

- to assist in planning and revising to maximise the potential of the programme.

The evaluation questions, presented in Box 1, will be used as a framework for this formative evaluation.

The main aim of a formative evaluation is to provide suggestions for improving the programme under evaluation. Practical improvements which will strengthen the link between programme activities and outcomes will be provided in the evaluation.

\section{Research design Research approach}

A descriptive design was utilised for this evaluation. According to Babbie and Mouton (1998, p. 105), descriptive research is the precise measurement and reporting of the characteristics of some population or phenomenon under study'. Primary data were collected by means of structured interviews with data providers.

\section{Research method}

\section{Research setting}

The evaluation took place in a large retail fashion organisation in Cape Town. Access to stores and the availability of data providers were specific challenges of this setting.

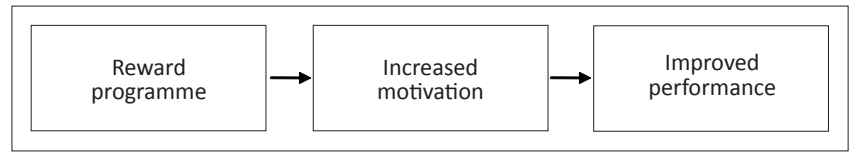

FIGURE 2: Programme theory.

BOX 1: Evaluation questions.

Question 1: Programme design

a. Is the change process assumed in the programme theory plausible?

b. Are employees satisfied with the reward being offered?

Question 2: Service delivery and resources

a. Are the procedures for identifying members of the target population, delivering the programme to them and sustaining the programme through to completion, well-defined and feasible?

b. Are the programme activities well-defined and sufficient to achieve the intended outcomes?

c. Are adequate resources allocated to the programme?

Question 3: Programme outcome

a. What are the programme's intended outcomes and are they well defined and feasible?

TABLE 1: Interview schedule.

\begin{tabular}{|c|c|c|}
\hline Evaluation questions & Interview questions & Data providers \\
\hline \multirow[t]{2}{*}{ Programme design } & $\begin{array}{l}\text { - Is the change process assumed } \\
\text { in the programme theory } \\
\text { plausible? } \\
\text { Do you feel the employees are } \\
\text { satisfied with the reward being } \\
\text { offered? }\end{array}$ & HRD Facilitator \\
\hline & $\begin{array}{l}\text { - Do you know what the reward } \\
\text { for the programme is? } \\
\text { - Are you satisfied with it? }\end{array}$ & Employees \\
\hline \multirow[t]{2}{*}{$\begin{array}{l}\text { Programme delivery and } \\
\text { resources }\end{array}$} & $\begin{array}{l}\text { - Are the procedures for } \\
\text { delivering the programme well- } \\
\text { defined? } \\
\text { - Are the procedures for } \\
\text { identifying the winners well- } \\
\text { defined? } \\
\text { - Are the programme activities } \\
\text { well-defined and sufficient to } \\
\text { achieve the programme goals? }\end{array}$ & $\begin{array}{l}\text { HRD Facilitator and } \\
\text { employees }\end{array}$ \\
\hline & $\begin{array}{l}\text { Do you believe the programme } \\
\text { resources are adequate? }\end{array}$ & HRD Facilitator \\
\hline Programme outcomes & $\begin{array}{l}\text { - What are the programme's } \\
\text { intended outcomes? } \\
\text { - Are they well-defined and } \\
\text { sufficient? }\end{array}$ & $\begin{array}{l}\text { HRD Facilitator and } \\
\text { employees }\end{array}$ \\
\hline
\end{tabular}

HRD, human resource development.

\section{Entrée and establishing researcher roles}

The Human Resource Development (HRD) Facilitator granted the first evaluator access to the programme and its records. Six months into the evaluation, access to programme records was restricted. Whilst this is later discussed in more detail under Limitations, the data collection procedure was somewhat compromised both in terms of sample size and access to documentation. Nonetheless all available programme records and documentation related to the programme description and implementation were consulted. Programme records relating to incentives were not available to the evaluator.

\section{Sampling}

Two streams of data providers were chosen, namely the HRD Facilitator who represented the organisation and a sample of employees who were the recipients of the programme. For the latter convenience sampling was used. As a result of time and access constraints within the organisation a sample of ten recipients (five permanent team members and five casual staff, comprising seven women and three men) who qualified for the reward was chosen. 


\section{Data collection methods}

Structured survey interviews were conducted with the HRD Facilitator and the sample of employees.

In Table 1, the evaluation questions, interview questions and data providers are described.

\section{Recording of data}

All data providers were interviewed individually by the primary evaluator. Responses were tape-recorded and transcribed.

\section{Data analyses}

The evaluation questions provided the structured themes of the analysis. The responses of the programme recipients to the interview questions were analysed quantitatively (number of respondents) and qualitatively (the specific meaning provided), and then compared with the responses of the programme manager where applicable.

\section{Reporting}

The findings for each evaluation question were reported and discussed qualitatively using common thematic extraction. Direct quotations from the interviews were selected as supporting evidence, where claims were made regarding the fidelity of the programme implementation.

\section{Findings}

The results are reported in terms of the evaluation questions which were derived from the programme theory.

\section{Question 1: Programme design}

The HRD Facilitator was of the opinion that the programme theory was plausible, as the previous year's successful programmes were based on this theory. The quote below illustrates this:

... our previous success proves that the programme motivates staff, drives development, and helps to retain employees ... so yes, I do think that this programme motivates staff and improves their performance. This is definitely plausible. (HRD Facilitator)

As a stakeholder with a vested interest in the programme, the HRD Facilitator's enthusiasm and confidence were expected. She also responded positively to the question related to the employees' perception of the reward:

... employees love the incentive and are extremely motivated to win it ... we use the three day get-away to really treat them and make them feel special and previous winners have loved the trips ... (HRD Facilitator)

To test the plausibility of the programme theory further, the employees were asked whether or not they knew what reward was offered and if they were happy with the reward. All ten employees criticised the choice of reward. The following quotations support the idea that the reward is not meaningful to the recipients:
... they take you to Head Office and to see the sights ... I think that's pathetic. I can do that in my own spare time. I wouldn't want to win that ... what's to achieve? (Recipient 3)

... it's a get-away in Cape Town to do things I've already done. Can't they give us more options that we actually want to win? (Recipient 5)

... a three day get-away ... but to me Table Mountain is nothing special. Why can't they ask me what I want to do? (Recipient 6)

... the reward is three days in Cape Town. This is not motivating for people who live in Cape Town. (Recipient 8)

Why can't they offer you a better salary rather or allow you to apply for more senior positions? Look at how many people work there ... this is not a reward. (Recipient 10)

\section{Question 2: Service delivery and resources}

All ten employees agreed that the procedures for delivering the programme, identifying the winners and sustaining the programme were neither well-defined nor sufficient. This directly challenged the HRD Facilitator's response to this same question. Most employees responded that the programme had not been successfully delivered to them, as is evident from the following quotes:

... they don't identify anybody and to be honest, it's not delivered at all ... maybe it's because I worked the late shift that day ... (Recipient 1 )

... how can delivery be well-defined or sufficient if I don't know anything about it and I've been working there for 2 years now? (Recipient 4)

... nobody explained anything to me ... I sort of found out via the grapevine when I saw the chart on the wall ... (Recipient 9)

... when you start late in the year, I think they just forget about you ... (Recipient 10)

The sample of employees acknowledged that the programme was successfully launched, but not sustained after the launch. The following selected quotations support this:

... you only hear about the programme when it starts [in June] and never again and then you just see points magically appearing ... (Recipient 1$)$

... I know about the programme, but I don't understand how they award points. Every time your points increase, but I don't know why. They don't even give you feedback ... (Recipient 2)

Another delivery question dealt with programme activities and their role in achieving outcomes. Again, the HRD Facilitator was positive about this aspect of the programme:

... these activities are drawn up to help employees perform more effectively and have been quite successful over the years ... (HRD Facilitator)

Six employees agreed that the activities were well-defined and easy to do. However, they were negative about the relevance of these activities to their performance. The following quotations were selected to illustrate these opinions:

... the activities make you look stupid. They give you these MCQs with nonsensical options. The [workbooks] are forced on you, but every year they ask the same questions. I don't see how that will make me a top performer ... so no, they are not sufficient to achieve the goals. (Recipient 3 ) 
... the activities don't make any sense. Staff don't even fill in the books and yet they get points. How is that supposed to achieve anything? (Recipient 5)

... the activities are stupid and pointless and only achieve one thing - undermining us ... (Recipient 7)

... nobody has time to fill in these books especially because they're unrelated to the goals ... (Recipient 10)

Four employees indicated that the activities did achieve the outcome of motivating staff, but that this was dependent on staff themselves. The following responses capture this sentiment:

... they could be sufficient to achieve the outcomes, but that depends on the person completing the activities ... (Recipient 1)

... I enjoy the activities because it really helps to refresh me about policies and procedures that I may have forgotten ... (Recipient 6)

... they can help to achieve the goals if you complete the [workbooks] as you should. It helps me. (Recipient 9)

The final delivery question dealt with adequacy of resources for programme implementation and was directed at the HRD Facilitator. She emphasised that resources, in terms of time, money, training and delivery of the programme were indeed adequate:

... we have allocated more than enough time and finances for the programme. We hold training workshops to train store and area managers on how to deliver the programme and a lot of money is spent on marketing the programme. We have a huge function every year at Head Office to introduce it and it has been extremely successful so far. (HRD Facilitator)

The HRD Facilitator's positive response could not be assessed against programme records relating to budget resulting from confidentiality issues raised by the organisation.

\section{Question 3: Programme outcomes}

Sufficient consensus existed regarding programme outcomes. The HRD Facilitator and seven employees agreed that improved customer service was an intended outcome. Three employees were uncertain of the outcomes, and their responses ranged from self-development, lowering pilfering to self-management.

Eight out of ten employees reported that the outcomes were not well defined and the following selected responses illustrate their disagreement:

... not at all ... I've never been told about how the programme works, what its outcomes or incentives are ... (Recipient 3)

... not all of it because most of the things they award you for, you don't even know where it comes from ... (Recipient 4)

... no ... nobody explains anything ... you just see a chart on the wall ... (Recipient 5)

Contrary to this, the HRD Facilitator and two employees considered that the outcomes are indeed well-defined:

... the programme's outcomes are well-defined and it has been running successfully for a few years ... (HRD Facilitator)

... well-defined? I think they are ... (Recipient 7)
In terms of the feasibility of outcomes all ten employees and the HRD Facilitator agreed that it was possible to achieve the outcomes. However, specific concerns were raised regarding their achievability:

... they are feasible but they change the rules all the time to try and make it harder for you to reach those goals ... (Recipient 1 )

... they would be feasible if the incentive was good enough ... (Recipient 6)

... they could be achieved if we knew for definite what they were ... (Recipient 9)

\section{Ethical considerations}

The Ethics in Research Committee of the Faculty of Commerce, University of Cape Town, approved the evaluation.

\section{Potential benefits and hazards}

There were no benefits or hazards for participants who took part in the evaluation. The HRD Facilitator agreed to be identified by role and the recipients of the programme who were interviewed remained anonymous.

\section{Recruitment procedures}

The HRD Facilitator granted the primary evaluator access to relevant programme documents and participants.

\section{Informed consent}

The primary evaluator explained the purpose of the interview and the time it would take to all recipients and the HRD Facilitator.

\section{Data protection}

The primary evaluator tape-recorded and transcribed all interviews. The primary evaluator was the only person who could access the transcriptions.

\section{Trustworthiness Reliability}

A structured survey was developed for the interviews (see Table 1).

\section{Validity}

The evaluation focused on a specific programme and did not seek to generalise the findings to other, similar programmes.

\section{Discussion}

The main objective of the evaluation was to explore whether or not this reward and recognition programme delivered its intended outcomes of increased motivation and improved performance. This evaluation contributes to the sparse literature that questions whether or not reward and recognition programmes are effective. 
The discussion will be presented in terms of the evaluation questions stated earlier plus the standards identified for successful reward and recognition programmes.

\section{Question 1: Programme design}

Whilst the HRD facilitator was of the opinion that the programme theory was plausible (in other words, that the programme led to increased motivation and improved performance), the plausibility was tested further by asking the employees whether or not they knew which reward was offered and if they were happy with it. All employees were critical of the reward. Whilst the causal theory may indeed be plausible, the responses from the sample of employees suggested that one of the elements necessary for a successful programme, namely meaningful rewards (Armstrong, 2002; DeWitt, 2001; Morrall, 1996), was absent. Such a reward or incentive may be either intrinsic or extrinsic, but should be meaningful to the recipient (Jensen et al., 2007). From the responses the sample appeared to show disdain for the ultimate reward, which is a key programme design flaw. In addition there was no clear consensus about the exact characteristics of the incentive. Although most of the sample group was aware that the reward was a three-day get-away in Cape Town, not many of their responses reflected confidence as to what this get-away entailed.

In summary, whilst one of the organisational stakeholders (the HRD Facilitator) viewed the programme theory as plausible and the reward as meaningful to the recipients, the data collected from the recipients were not aligned with this opinion. This evidence suggested that the programme theory and design might be weak and inconsistent. A proper needs analysis may generate more meaningful rewards which could be more motivating.

\section{Question 2: Service delivery and resources}

In terms of programme delivery and sustainability it was clear that the programme has not been implemented as intended. This was evident from the negative responses from all ten employees who criticised these two aspects of the programme. In addition, those employees who were familiar with the programme did not seem to understand the way the programme was intended to work. The basis of the programme (a points system) did not appear to be welldefined or maintained. Employees were not aware of how points were awarded. This lack of knowledge is a concern. It was also apparent that whilst some employees initially received the programme, delivery of the programme was not being sustained through to completion. The programme was intended to target all staff. However, delivery inconsistencies suggested that this was not the case.

The majority of employees agreed that the programme activities were well defined and easy to perform. However, they questioned the relevance of the activities to improved performance. These responses showed a marked difference to the response of the HRD Facilitator who postulated a clear link between programme activities and improved performance. Furthermore, some employees indicated that staff motivation depended on individual employees and not on the programme.

The HRD Facilitator indicated that there were sufficient resources for programme implementation. However, this response could not be assessed against programme records.

In summary, service delivery did not happen as intended. The programme seemed to have been launched effectively, but not sustained to completion. The programme was not fully understood by its target audience and some of the programme activities (e.g. the workbook completion) were not completed as intended. Furthermore, some of the activities were regarded as meaningless and as not contributing to improved performance. These programme delivery shortcomings may have a serious influence on the effect of the programme.

\section{Question 3: Programme outcomes}

From the results it was evident that most of the sample agreed with the HRD Facilitator that one intended outcome of the programme was to improve customer service. However, a minority identified other outcomes, such as self-management and the prevention of stock loss and theft. This may become problematic as not all recipients might be working towards the same behaviour change that the programme was supposed to bring about.

Whilst there was consensus about the outcome of improved customer service most employees agreed that the definition of the outcomes was not sufficiently clear. Responses suggested that the outcomes were not discussed with employees and, whilst the initial roll-out might have been satisfactory, programme delivery had been poor, affecting the programme's outcomes. This was supported by the available programme documents, which failed to clarify programme outcomes. From the literature (Jensen et al., 2007; Zenger, 1992) it was clear that understanding outcomes is an important step in the process of achieving them. Lack of such understanding could confuse recipients and fail to direct their behaviour to achieving the intended outcomes.

In summary, there seemed to be sufficient consensus that the programme was intended to improve customer service. However it is questionable whether or not all outcomes were clearly defined and understood.

\section{Evaluating against the standard for reward programmes}

Reward programmes, which recognise that employees need to value the reward, tend to be successful (Morrall, 1996). From the employees' responses it became clear that none of the sampled recipients valued the reward or were motivated by it to improve their performance. Some responses indicated that recipients would prefer intrinsic 
rewards (e.g. the opportunity to develop their careers in the organisation), or more relevant extrinsic rewards (e.g. increased pay). These suggest that recipients are at varying stages of need and motivation and that a programme which does not take this into account may even decrease employees' motivation levels. A standard reward that is not valued may appear manipulative and may undermine the effect of the programme.

From the data it was unclear what organisational culture is like and whether it supports a customer service-focused reward programme or not. Judging from the responses, indicating the lack of clarity regarding the outcome of the programme, it seemed as if clear channels of communication do not exist at all levels of the organisation. This was reinforced by the maturity of the programme: it has been running for five years and yet recipients are still unaware of the rewards and the outcome of the programme.

The programme design shows that it is aligned with strategic objectives as it is intended to improve customer service, increase motivation and reduce turnover. For a retail business, these objectives are relevant, realistic and geared towards greater organisational success.

Best practice performance indicators should be clear and unambiguous (Benardin, 2003). From the data it could be concluded that recipients were aware of how points can be earned, but were uncertain as to how these points are allocated. There were niggling indications that the technical details of the point system are not understood (e.g. only smaller stores achieve the rewards, unfair and inconsistent awarding of points, etc.). The lack of clear and unambiguous corporate goals and performance indicators could prevent employees from striving towards common goals and thus undermine the programme outcome (Jensen et al., 2007; Zenger, 1992).

The recipients of successful reward programmes believe that excellent performance will lead to rewards. The recipients of this programme were not confident that this is the case. This is a serious flaw of the programme implementation. If reward is perceived to be distributed arbitrarily or if recipients are of the opinion that only people in specific stores receive the reward, then a disjuncture exists between performance and reward, and this may affect employee motivation negatively.

Whilst the programme is based on specific indicators like turnover, customer feedback and the number of new accounts opened, no standards are supplied for these indicators. Recipients know that they have to work towards these indicators, but do not know how much is good enough to earn the award. In the literature (Benardin, 2003; Jensen et al., 2007; Zenger, 1992), there is agreement that indicators and standards have to be focused, reasonable, measurable, participatory, neutral, challenging, achievable and should provide the basis for feedback. These indicators and standards may well be present in the programme records (the evaluators had restricted access to this). If so, failure to communicate this to recipients may undermine the effect of the programme.

Out of the six guidelines for successful reward programmes, it would seem that the programme under evaluation conformed to one, namely programme goals aligned with organisational strategy. The evaluators recommend that stakeholders improve the programme theory and design, based on the research and results presented here.

\section{Conclusion}

From the findings it was clear that the HRD Facilitator and the recipients had different opinions regarding the plausibility of the programme theory and the value attached to the rewards. Furthermore, the evaluators found inconsistencies in programme delivery and sustainability across sites. On the other hand, both the HRD Facilitator and the recipients agreed that the programme improved customer service. This finding was important, as it showed that the programme goals were aligned with the organisation's strategy.

\section{Recommendations}

Reward programmes affect motivation and performance (Cameron \& Pierce, 1996; Eisenberger \& Cameron, 1996; Nelson, 1998; Platten, 1996). Therefore, the evaluators suggest that the programme theory be reviewed in order to generate a plausible theory, which would contain all the causal links postulated by the HRD Facilitator. It may look like the diagram in Figure 3.

From this limited evaluation, it is clear that some programme components may unintentionally undermine the proposed theory. In order to improve this, programme activities should be developed in alignment with the revised programme theory.

A second recommendation is to conduct an ongoing and formal needs analysis to ascertain which rewards are valued by the recipients of the programme. From the evaluation, it was clear that the recipients were not enthralled by a weekend in Cape Town. Valued rewards would also address the rather abstract question of what motivates people, and highlight that some recipients choose extrinsic and others intrinsic rewards as motivators.

Thirdly, a systematic programme delivery process could ensure that all recipients understand the different aspects

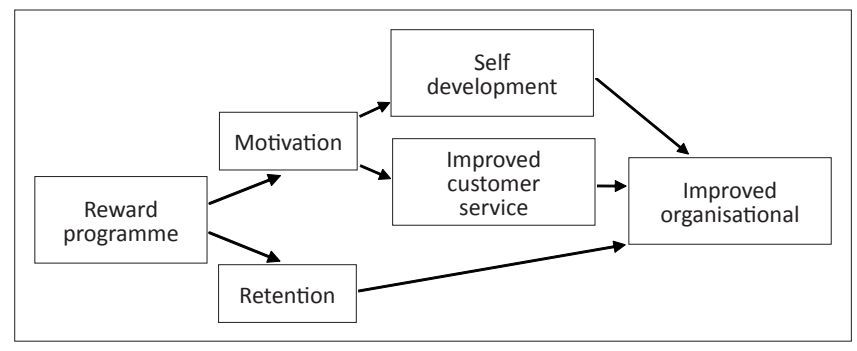

FIGURE 3: Plausible programme theory for a reward programme. 
of the programme (theory, outcomes, indicators, standards, process of awarding points, etc.). This delivery process could be evaluated in order to ascertain whether or not it has resulted in increased consensus and understanding of the programme.

Fourthly, the programme needs to clarify performance indicators and standards to ensure that recipients understand what they are striving for and what level of performance is good enough. This should foster support for the programme and contribute to its success.

A final recommendation is an ongoing monitoring process to track whether or not programme outcomes are being achieved and implementation processes followed. The information from this process could be used in a formative manner, to improve the programme on an annual and ongoing basis.

\section{Limitations}

Firstly, six months into the evaluation, the evaluators were required to sign a confidentiality clause. This resulted in restrictions being placed on the provision of relevant financial data and free access to programme records. These restrictions precluded specific aspects of an implementation evaluation.

Secondly, the evaluation was limited by the non-availability of specific data providers. The busy schedule of the HRD Facilitator affected the quality of the information relating to the programme description and theory.

Thirdly, the small sample group who partook in the evaluation may not have been representative of the intended target population. The evaluators were only able to source recipients from two stores. However, the ten recipients provided valuable data regarding their experience of the programme and these data were useful when comparing the current programme against standards of good practice for reward programmes.

\section{Future research}

This evaluation highlighted an important evaluation experience, namely how to work around unexpected restrictions placed on access to programme records. Firsttime evaluators often expect unlimited access to clean, abundant data. This may not be the case and evaluators need to understand how to cope with hindrances and obstacles in their way to a good evaluation.

This evaluation contributed to the limited literature on the effect of reward programmes. Whilst there is a vast amount of literature pertaining to reward programmes, very few evaluations of such programmes exist. This evaluation provided the opportunity to ask: 'How does the programme work'; and 'Does the programme work?' Continuing this line of inquiry in future research may raise the level of debate regarding the effects of reward programmes.

\section{Acknowledgements Competing interests}

The authors declare that they have no financial or personal relationship(s) which may have inappropriately influenced them in writing this paper.

\section{Authors' contributions}

S.S. (University of Cape Town) completed this evaluation as a requirement for her Master's degree in Programme Evaluation. A.S. (University of Cape Town) supervised the evaluation.

\section{References}

AccelTeam. (2008). Employee motivation: Theory and practice. Retrieved October 17, 2008, from http://accel-team.com/motivation/

Amabile, T.M. (1993). Rethinking rewards. Harvard Business Review, 71(6), 37-49.

Applebaum, E. (1993). Rethinking rewards. Harvard Business Review, 71(6), 37-49.

Armstrong, M. (2002). Employee reward. (3rd edn.). London: Chartered Institute of Personnel Development.

Babbie, E., \& Mouton, J. (1998). The practice of social research. Oxford: Oxford University Press.

Baker, G.P. (1993). Rethinking rewards. Harvard Business Review, 71(6), 37-49.

Beer, M. (1993). Rethinking rewards. Harvard Business Review, 71(6), 37-49.

Benardin, H.J. (2003). Human resource management: An experiential approach. Boston: McGraw-Hill.

Bishop, J. (1986). The recognition and reward of employee performance. Retrieved November 23, 2008, from http://www.eric.ed.gov/ERICDocs/data/ericdocs2sql/ content_storage_01/0000019b/80/2f/32/de.pdf

Bhola, H.S. (1990). Evaluating 'Literacy for development' projects, programs and campaigns: Evaluation planning design and implementation and utilization of evaluation results. Hamburg: UNESCO Institute for Education.

Bowen, R.B. (2000). Recognizing and rewarding employees. New York: McGraw Hill.

Cameron, J., \& Pierce, W.D. (1996). The debate about rewards and intrinsic motivation: Protests and accusations do not alter the results. Review of Educational Research, 66(1), 39-51.

Chappuis, S., \& Chappuis, J. (2008). The best value in formative assessment. Educational Leadership, December 2007 - January 2008, 14-18.

Chen, H.-T. (2005). Practical program evaluation: Assessing and improving planning implementation and effectiveness. Thousand Oaks: Sage.

Deci, E.L., \& Ryan, M.R. (1985). Intrinsic motivation and self-determination in human behaviour. New York: Springer.

Denning, S. (2001). Incentives for knowledge management. Incentives for Knowledge Management - Incentives for Knowledge Sharing 2000. Retrieved n.d., from

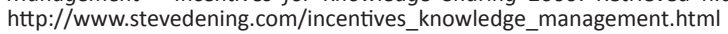

DeWitt, K. (2001). Incentives for profit. Commercial Carrier Journal, 158(10).

Eisenberger, R., \& Cameron, J. (1996). The detrimental effects of reward: Myth or reality? American Psychologist, 51, 1153-1166. http://dx.doi.org/10.1037/0003066X.51.11.1153, PMid:8937264

Harrison, D.A., Virick, M., \& William, S. (1996). Working without a net: Time, performance, and turnover under maximally contingent rewards. Journa of Applied Psychology, 81(4), 331-345. http://dx.doi.org/10.1037/0021of Applied Psyc

Herzberg, F. (1959). The motivation to work. New York: Wiley/Chapman \& Hall.

Jensen, D., McMullen, T., \& Stark, M. (2007). The manager's guide to rewards. Philadelphia: Hay Group, Inc.

Kohn, A. (1993). Why incentive plans cannot work. Harvard Business Review, 71(5), 54.

Morrall, K. (1996). Motivating sales staff with rewards. Bank Marketing, 28(7).

Mottaz, C.J. (1985). The relative importance of intrinsic and extrinsic rewards as determinants of work satisfaction. The Sociological Quarterly, 26(3), 365-385. http://dx.doi.org/10.1111/j.1533-8525.1985.tb00233.x

Nelson, B. (1998). Influencing top management on the value of incentive programs. Fresno Business Journal, 322-349.

Patton, M. (1997). Utilization-focused evaluation. (3rd edn.). Thousand Oaks: Sage.

Platten, P.E. (1996). Incenting with a vengeance. CFO, 12(6). 
Rossi, P., Lipsey, M.W., \& Freeman, H.E. (2004). Evaluation. A systematic approach (7th edn.). Thousand Oaks: Sage.

Rummel, A., \& Feinberg, R. (1988). Cognitive evaluation theory: A meta-analytic review of the literature. Social Behavior and Personality, 16, 147-164. http:// dx.doi.org/10.2224/sbp.1988.16.2.147

Rusbult, C.E., \& Farrell, D. (1983). A longitudinal test of the Investment Model: The impact on job satisfaction, job commitment, and turnover of variations in rewards, costs, alternatives, and investments. Journal of Applied Psychology, 68(3), 429-438. http://dx.doi.org/10.1037/0021-9010.68.3.429

Ryan, R.M., \& Deci, E.L. (2000). Self-determination theory and the facilitation of intrinsic motivation, social development, and well-being. American Psychologist, 55(1), 68-78. http://dx.doi.org/10.1037/0003-066X.55.1.68, PMid:11392867

Tang, S.-H., \& Hall, V.C. (1995). The overjustification effect: A meta-analysis. Applied Cognitive Psychology, 9, 365-404. http://dx.doi.org/10.1002/acp.2350090502
Trochim, W.M.K. (2006). Introduction to evaluation. Research Methods Knowledge Base. Retrieved n.d., from http://anatomyfacts.com/Research/ ResearchMethodsKnowledgeBase.pdf

Ulrich, D., \& Brockbank, W. (2005). The HR value proposition. Boston: Harvard Business School Press.

Vroom, V.H. (1964). Work and motivation. New York: John Wiley \& Sons.

Wiersma, U.J. (1992). The effects of extrinsic rewards in intrinsic motivation: A metaanalysis. Journal of Occupational and Organizational Psychology, 65, 101-114. http://dx.doi.org/10.1111/j.2044-8325.1992.tb00488.x

Zachary, W.B., \& Kuzuhara, W.L. (2005). Organizational behaviour: Integrated models and applications. Mason: Thomson.

Zenger, T. (1992). Why do employers only reward extreme performance? Examining the relationships among performance, pay, and turnover - process and outcome:
Perspectives on the distribution of rewards in organisations. Administrative Science Quarterly, 37, n.p. 